\title{
Heterodyne Detection of Optical Terahertz Beat-Tones Based on Semiconductor Optical Amplifier
}

\author{
Séverine Philippe, Ramon Maldonado-Basilio, \\ and Pascal Landais, \\ Sylwester Latkowski, \\ The RINCE Institute, Dublin City University, Dublin 9, Ireland
}

\begin{abstract}
In this paper, a detection scheme based on four wave mixing in a semiconductor optical amplifier (SOA) is used to measure the frequency and bandwidth of the optical terahertz beat-tone signal generated by a passively mode locked semiconductor laser. Two signals from external cavity lasers are injected into an SOA, which acts as a local oscillator at their detuning frequency. The device under test is a passively mode locked laser with a beattone frequency in the terahertz spectrum. Its optical signal is injected in the SOA, which also acts as a frequency mixer. The frequency and linewidth of the terahertz signal are then measured by retrieving the intermediate frequency detected by a low bandwidth photodiode and an electrical spectrum analyser.
\end{abstract}

\section{INTRODUCTION}

The terahertz spectrum, from $300 \mathrm{GHz}$ to $10 \mathrm{THz}$ has many applications, such as medical imaging, manufacturing and security screening. However schemes available for generation and detection of these waves are still limited.

Intracavity mode beating in DC biased multimode Fabry-Pérot semiconductor laser has been demonstrated as an easy way to generate optical signals modulated at a radio-frequency corresponding to the mode detuning [1]. Recently, we have demonstrated that the gain dynamics in multimode laser can sustain $\mathrm{THz}$ optical beat-tones [2-3]. In our approach, the $\mathrm{THz}$ beat-tones generated benefit from a good overlap between the spatial modes and also from the good stability of the detuning as modes undergo the same intra-cavity fluctuations. Compared to other $\mathrm{THz}$ generation schemes, our technique does not require any external source of modulation or cryogenic temperature control. Therefore, this solution is more stable and its footprint is smaller.

Characterization of $\mathrm{THz}$ sources are usually achieved by using bolometers, Golay cells and pyroelectric detectors [4,5]. Other schemes have been proposed, analyzing the RF spectrum of light sources by means of optical techniques such as nonlinear interactions in highly non-linear fibers [6] and cross-phase modulation in nonlinear waveguides [7]. In spite of the great achievements accomplished in the majority of the mentioned schemes in terms of frequency resolution, in the order of $\mathrm{GHz}$, and bandwidth (from $100 \mathrm{GHz}$ to few $\mathrm{THz}$ ), their main drawbacks are the large experimental set-ups used and the cost of the necessary components.

On the other hand our approach, which can be used for two-color [8] and multimode semiconductor lasers, is simpler and also allows access to the bandwidth of the signal with increased resolution: we present an investigation of the $\mathrm{THz}$ beat-tones in a multimode semiconductor laser using a semiconductor optical amplifier (SOA) under four-wave-mixing (SOA-FWM) acting as mixer/local oscillator for a heterodyne detection of terahertz signals.

\section{DEVICE UNDER TEST}

The device under test (DUT) is a $\sim 700 \mu \mathrm{m}$ long multi-quantum well InAlGaAs Fabry-Perot (FP) laser, with a one-dimensional photonic band-gap embedded in its structure [9]. This photonic band-gap is realized by etching a number of shallow grooves on the p-side of the laser junction, perpendicular to the longitudinal axis of the device. The resulting variation in the refractive index experienced by the electromagnetic wave generated in the active layer allows us to control the longitudinal spectrum of the device.

In this particular device, the FP resonance determined by the length of the device results in a $\sim 0.5 \mathrm{~nm}$ modulation, as can be seen on the inset of Fig. 1(a). On the other hand the etched band-gap suppresses or enhances some specific FP modes, resulting in our case in a $2.5084 \mathrm{~nm}$ free spectral range.

The DUT is not packaged and is therefore tested using a probe station. It is a single-section device and the cavity does not feature any saturable absorber. Its optical output current dependence is shown on Fig. 1(b): its threshold current is $18 \mathrm{~mA}$ and the maximum collected output power $1.2 \mathrm{~mW}$. During the following experiment the DUT is DC biased at $90 \mathrm{~mA}$ and temperature controlled at $25^{\circ} \mathrm{C}$. The multimode characteristic of the device is clearly apparent on its spectrum, as can be seen on Fig. 1(a). The device output power at this bias current is $1.1 \mathrm{~mW}$. 


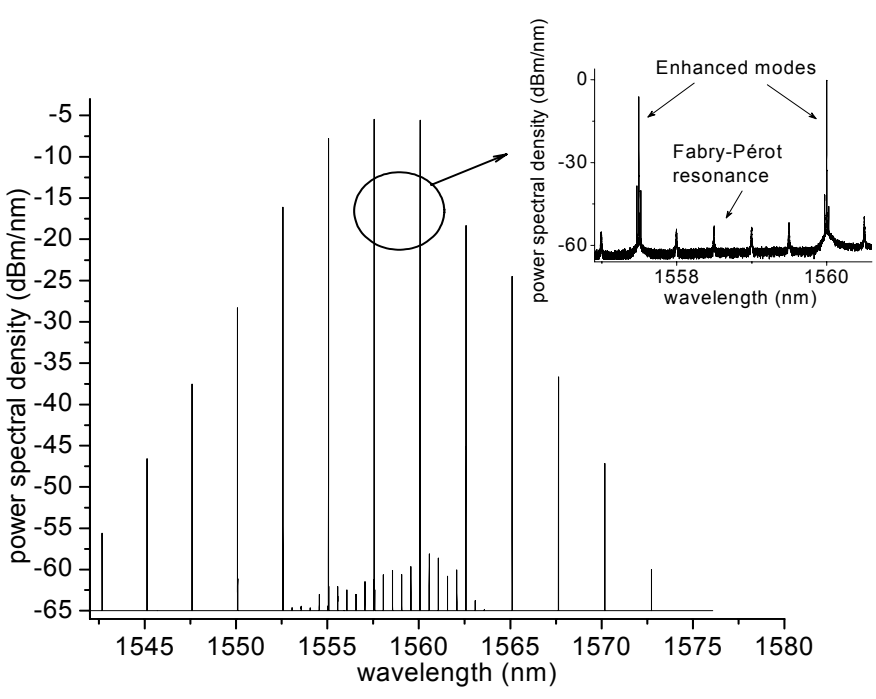

Figure 1(a). Spectrum of DUT at $I_{\text {bias }}=90 \mathrm{~mA}$, inset: spectrum showing two main modes and Fabry-Pérot resonances.

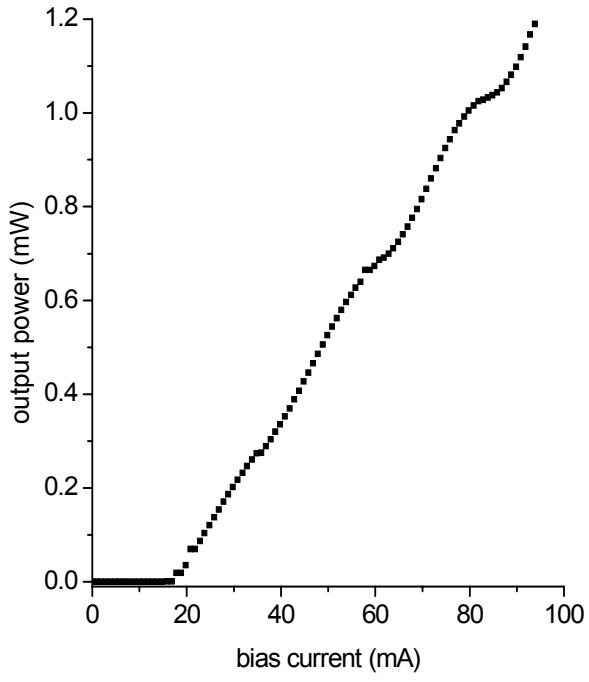

Figure1(b). LI curve of DUT.

\section{EXPERIMENT}

\subsection{Principle}

A wave mixing experiment is implemented, where signals from the DUT as well as two tunable external cavity lasers (ECL) are injected into a SOA. An optical isolator is used to avoid feedback into the DUT and each of the three input arms are polarisation controlled, before being amplified with EDFAs. The output of the SOA is then monitored using an optical spectrum analyser (OSA) or a photodiode connected to an electrical spectrum analyser (ESA). The SOA is a high confinement factor ultra-fast non-linear device with low polarization dependence. There exist multiple beating processes inside the SOA between the input beams and their corresponding conjugated and engendered signals. However the two main processes of interest are described below.

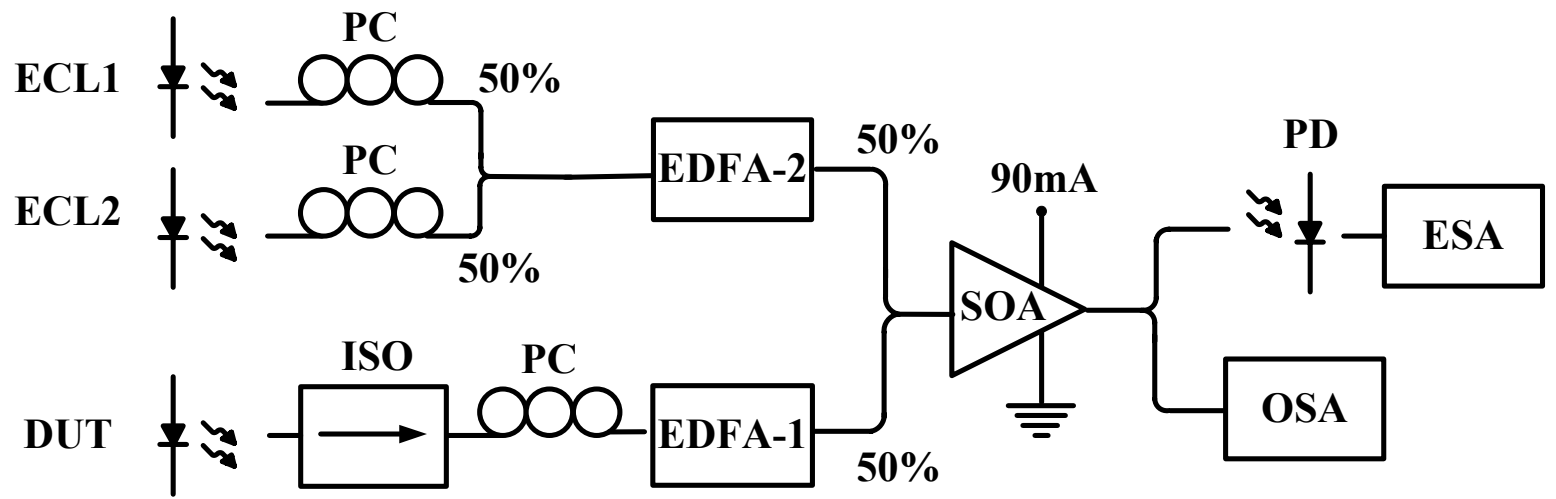

Figure 2. Experimental set-up.

Under injection of the two ECL signals, four wave mixing (FWM) takes place in the SOA. Thus the SOA operates as a local oscillator whose frequency $\Delta f_{\mathrm{ECL}}$ is tunable and given by the difference $c\left|1 / \lambda_{\mathrm{ECL} 1}-1 / \lambda_{\mathrm{ECL} 2}\right|$, where $c$ is the speed of light in vacuum, and $\lambda_{\mathrm{ECL}}$ the wavelength of the ECL. There is a modulation of the complex refractive index (gain and refractive index) of the active material at the frequency $\Delta f_{\mathrm{ECL}}$. The modulation frequency can be changed by varying the detuning between the ECL wavelengths. This also changes the index of the modulation, depending on the efficiency of the FWM processes selected by the detuning.

In addition, mixing between the DUT signal and the local oscillator frequency results in beating at an intermediate frequency $\Delta f_{\mathrm{IF}}=\left|\Delta f_{\mathrm{DUT}}-\Delta f_{\mathrm{ECL}}\right|$, where $\Delta f_{\mathrm{DUT}}$ is the frequency to be resolved. It is important to note here that while $\Delta f_{\mathrm{DUT}}$ and $\Delta f_{\mathrm{ECL}}$ may be in the range of several GHz to a few THz, their difference $\Delta f_{\mathrm{IF}}$ can be much lower. Indeed the choice of the ECLs frequencies is crucial and must take into account the bandwidth of the photodiode detector. 


\subsection{Results}

For demonstration purposes, the SOA output is monitored with an OSA and different components can be observed on its spectrum, as shown on Fig. 3(a). One set corresponds to the DUT signal after injection and nonlinear amplification in the SOA, where its main modes are enhanced, giving rise to intra-band carrier-heating dynamic at $\Delta \mathrm{f}_{\text {DUT }} \approx 310 \mathrm{GHz}$. The two ECLs wavelengths are set to $1539.0099 \mathrm{~nm}$ and $1536.5406 \mathrm{~nm}$ respectively so that their non-linear beating inside the SOA generates two main side bands at $\left(\lambda_{\mathrm{ECL} 1}-c / \Delta f_{\mathrm{ECL}}\right)$ and $\left(\lambda_{\mathrm{ECL} 2}+c / \Delta f_{\mathrm{ECL}}\right)$. In our case this gives rise to an intra-band carrier heating dynamic at $\Delta f_{\mathrm{ECL}} \approx 313.26 \mathrm{GHz}$. It is important to note here that the modulations due to injection of the DUT and ECLs signals arise not only at frequencies $\Delta f_{\mathrm{DUT}}$ and $\Delta f_{\mathrm{ECL}}$, which are in the terahertz spectrum, but also at their difference $\Delta f_{\mathrm{IF}}=\left|\Delta f_{\mathrm{DUT}}-\Delta f_{\mathrm{ECL}}\right|$, a much lower frequency. The peaks arising from the modulation at $\Delta f_{\mathrm{ECL}}$ can be seen beside each of the components previously described and are shown on the inset of Fig. 3(a) as well as the intermediate frequency. The wavelengths of the ECLs are chosen so that the intermediate frequency $\Delta f_{\mathrm{IF}}$ is within the detector bandwidth, in our case of $10 \mathrm{GHz}$.

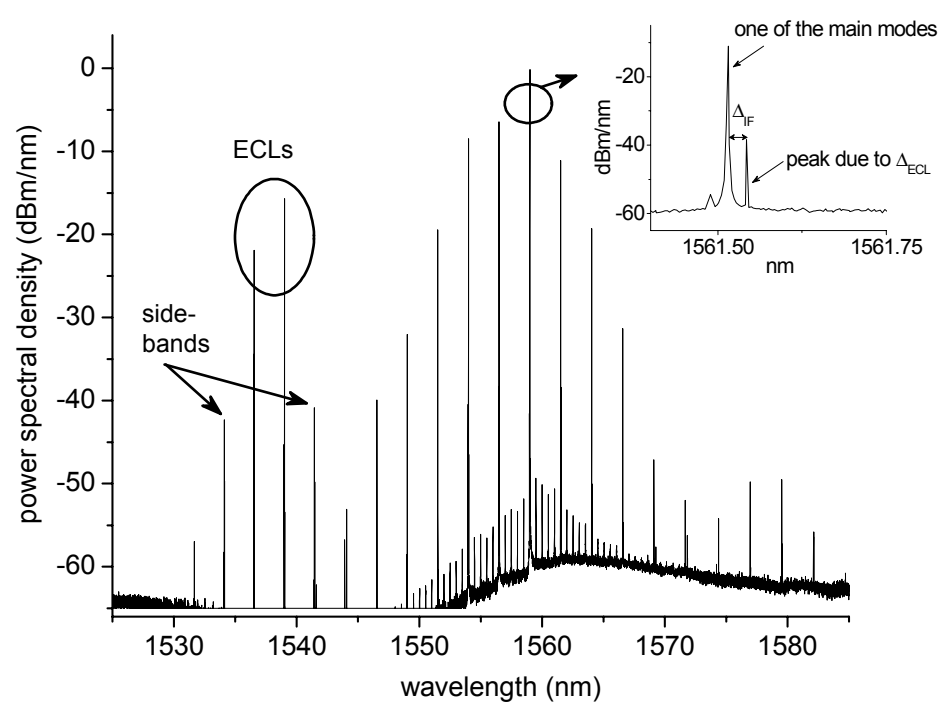

Figure 3(a). DUT spectrum, under injection. $I_{\text {bias }}=90 \mathrm{~mA}$ $\lambda_{E C L 1}=1539.0099 \mathrm{~nm}, \lambda_{E C L 2}=1536.5406 \mathrm{~nm}$.

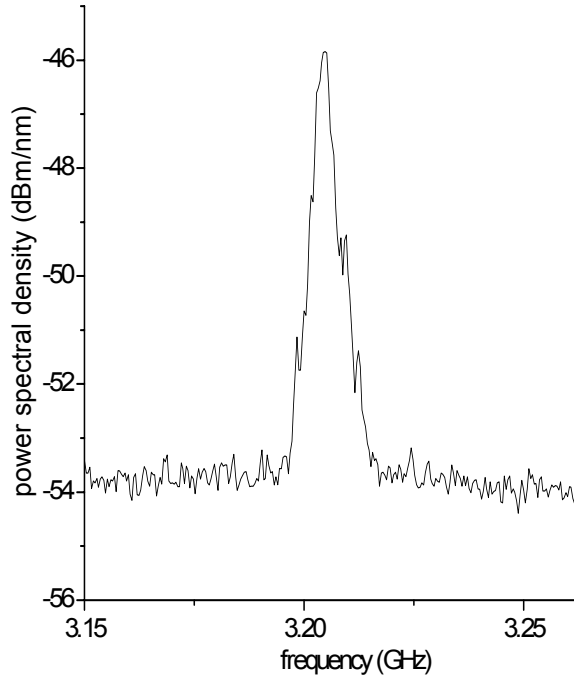

Figure 3(b). Corresponding ESA trace for intermediate frequency.

By measuring $\Delta f_{\mathrm{IF}}$ at the output of the SOA, using a widely available $10 \mathrm{GHz}$ photodiode connected to an ESA, the modulation frequency of the DUT can be found. The RF spectrum of the SOA output, Fig. 3(b), shows a centre frequency of $3.205 \mathrm{GHz}$, allowing the modulation frequency of the DUT to be determined. It was found that $\Delta f_{\mathrm{DUT}}=\Delta f_{\mathrm{ECL}}-\Delta f_{\mathrm{IF}} \approx 310.05 \mathrm{GHz}$. The limiting factor for the accuracy of $\Delta f_{\mathrm{DUT}}$ is the OSA resolution, which is $0.16 \mathrm{pm}$ or $\sim 20 \mathrm{MHz}$. Therefore $\Delta f_{\mathrm{DUT}}=310.05 \pm 0.02 \mathrm{GHz}$. In addition, the full width half maximum (FWHM) of the IF signal is measured as $8 \mathrm{MHz}$ when fitted to a Lorentzian lineshape. Since the FWHM linewidth of each ECL is of the order of $100 \mathrm{kHz}$, their contribution to the linewidth of the IF signal is about $2.5 \%$ and the linewidth of the signal is properly expressed by $8 \pm 0.2 \mathrm{MHz}$. Furthermore, the sum of the optical FWHM linewidths of the modes was found to be $\sim 377 \mathrm{MHz}$, lager than that of the beat-tone measured, thus showing that passive mode locking is taking place inside the DUT.

It is possible to vary the detuning of the ECLs, thus varying the frequency of the SOA acting as a local oscillator. It is therefore possible to achieve a tunability of the local oscillator within the RF and the THz band. In addition to the optimal resolution of the OSA and ESA used, the limiting factors of the technique in the low portion of the RF band is the stability and the resolution of the ECL emission, and the maximal value of the local oscillator frequency is determined by the characteristic lifetime of the spectral-hole-burning in the SOA. It has been demonstrated to be of the order of $10 \mathrm{THz}$ [11], so within the $\mathrm{THz}$ band.

\section{CONCLUSIONS}

In conclusion $\mathrm{THz}$ beat-tones generated by a passively mode-locked laser were resolved using top of the shelf equipment. The experimental setup used for this measurement also operates at room temperature, and is capable of resolving optical $\mathrm{THz}$ beat-tone signals across both the $\mathrm{RF}$ and the $\mathrm{THz}$ bands. Both the frequency and linewidth of the terahertz signal were retrieved, with an error $20 \mathrm{MHz}$ and $0.2 \mathrm{MHz}$, respectively.

The detection technique uses a SOA acting both as a local oscillator, at the carefully chosen detuning frequency between two ECL signals, and as a frequency mixer for these two signals and the output of the DUT. 
The frequency and linewidth of the DUT signal is then retrieved from the intermediate frequency, which is measured using a $10 \mathrm{GHz}$ photodiode and an ESA. This method constitutes a simple solution for precise measurement of $\mathrm{THz}$ beat-tones linewidth and is relatively easy to implement using only widely available components at room temperature.

\section{ACKNOWLEDGEMENTS}

Séverine Philippe thanks the Irish Research Council for Science, Engineering and Technology for their support.

\section{REFERENCES}

[1] K. Sato: $100 \mathrm{GHz}$ optical pulse generation using Fabry-Perot laser under continuous wave operation, Electronics Letters 37 (12), 763 (2001).

[2] S. Latkowski, F. Surre and P. Landais: Terahertz wave generation from a dc-biased multimode laser, Appl. Phys. Lett. 92 (8) (2008), p. 081109

[3] S. Latkowski, F. Surre, R. Maldonado-Basilio, P. Landais: Investigation on the origin of terahertz waves generated by dc-biased multimode semiconductor lasers at room temperature, Appl. Phys. Lett. 93 (24) (2008) 241110

[4] P. H. Siegel: Terahertz technology, IEEE Transactions on Microwave Theory and Techniques, vol. 50, no. 3 (2002).

[5] M. Naftaly, P. Dean, R. E. Miles, J. R. Fletcher, and A. Malacoci: A simple interferometer for the analysis of terahertz sources and detectors, IEEE J. Sel. Top. Quantum Electron. 14, 443 (2008).

[6] C. Dorrer and D. N. Maywar: RF spectrum analysis of optical signals using nonlinear optics, J. Lightwave Technol. 22, 266 (2004)

[7] M. Pelusi, F. Luan, T. D. Vo, M. R. E. Lamont, S. J. Madden, D. A. Bulla, D. -Y. Choi, B. Luther-Davies, and B. J. Eggleton: Photonic-chip-based radio-frequency spectrum analyser with terahertz bandwidth, Nat. Photonics 3, 139 (2009).

[8] S. Hoffmann, M. Hofmann, E. Bründermann, M. Havenith, M. Matus, J. V. Moloney, A. S. Moskalenko, M. Kira, S. W. Koch, S. Saito, and K. Sakai: Four-wave mixing and direct terahertz emission with twocolor semiconductor lasers, Appl. Phys. Lett. 84, 3585 (2004)

[9] P. Landais, Patent number S2005/0251

[10] S. Latkowski, R. Maldonado-Basilio, K. Carney, J. Parra-Cetina, S. Philippe, and P. Landais : Semiconductor optical amplifier-based heterodyning detection for resolving optical terahertz beat-tone signals from passively mode-locked semiconductor lasers, Appl. Phys. Lett. 97, 081113 (2010).

[11] A. D’Ottavi, E. Iannone, A. Meccozzi, S. Scotti, P. Spano, R. Dall'Ara, G. Guekos, and J. Ecker: Frequency conversion by four-wave mixing on a frequency range of $8.6 \mathrm{THz}$, in Proc. European Conference on Optical Communication, pp. 737-740 (1994). 